\title{
Estudo do tempo de processamento para análises do comportamento mecânico de córneas via simulações numéricas
}

\author{
Paulo Roberto de Almeida ${ }^{1}$ \\ Ricardo Rodrigues Magalhães ${ }^{2}$
}

\begin{abstract}
Resumo: A córnea e a esclera formam a superfície externa do globo ocular, proporcionando proteção aos componentes internos e mantendo o formato de refração do olho. A estrutura da córnea é composta de epitélio, endotélio e estroma, sendo este último o principal responsável pelas características mecânicas da córnea. Estudos biomecânicos envolvendo o Método dos Elementos Finitos (MEF) podem revelar características mecânicas importantes da córnea, porém estes estudos normalmente requerem alto custo computacional. Este trabalho propõe um estudo do tempo de processamento para análises do comportamento mecânico de córneas humanas via MEF, levando-se em consideração modelos computacionais com baixa complexidade. As simulações foram realizadas utilizando um software comercial de análise de elementos finitos para medir o deslocamento no ponto central da córnea, em que variou-se a quantidade de elementos e nós dos modelos, com o objetivo de comparar os resultados.
\end{abstract}

Palavras-chave: Biomecânica. Córnea. Método dos Elementos Finitos.

\begin{abstract}
Cornea and sclera are parts of the ocular globe external surface, providing protection to the internal components and keeping the shape of eye refractive. The corneal structure is composed by epithelium, endotheliumn and stroma, being this last one the main responsible for the mechanical properties of the cornea. Biomechanical studies involving the Finite Element Method (FEM) can reveal important mechanical characteristics of the cornea, however these studies usually require high computational cost. This work proposes a study of the processing time for mechanical behavior analysis of human corneas by using FEM, taking into consideration computational models with low complexity. Simulations were performed using a commercial software finite element analysis to measure the displacement at the central point of the cornea in which the amount of elements and nodes of the model was changed in order to compare the results.
\end{abstract}

\section{Keywords: Biomechanics. Cornea. Finite Element Method.}

\section{Introdução}

Uma das partes mais importantes do globo ocular é a córnea a qual possui características mecânicas que podem ser analisadas no intuito de aprimorar métodos para prevenção de doenças e o surgimento de equipamentos mais eficientes na área médica [1]. Ter uma compreensão do comportamento mecânico de córneas é importante para constatar aplicações clínicas, como exemplos: a tonometria, diagnósticos de ceratocone e estudos relacionados às cirurgias refrativas [2].

As propriedades biomecânicas da córnea humana estão relacionadas à sua estrutura, em especial ao comportamento do estroma [3]. A elasticidade da córnea depende da forma como as fibras de colágeno estão agrupadas, variando conforme a direção das fibras [4]. Estudos realizados sobre o comportamento da córnea humana demonstram comportamento elástico não-linear, tendo seu módulo de elasticidade (módulo de Young), crescente, à medida em que a tensão aplicada é aumentada [5].

\footnotetext{
${ }^{1}$ Programa de Pós-Graduação em Engenharia de Sistemas e Automação (PPGESISA), UFLA, Campus Lavras - Lavras (MG) - Brasil \{pralmeida22@gmail.com\}

${ }^{2}$ Programa de Pós-Graduação em Engenharia de Sistemas e Automação (PPGESISA), UFLA, Campus Lavras - Lavras (MG) - Brasil \{ricardormedeg.ufla.br\}
}

http://dx.doi.org/10.5335/rbca.v9i4.7025

Revista Brasileira de Computação Aplicada (ISSN 2176-6649), Passo Fundo, v. 9, n. 4, p. 32-42, dez. 2017 
Magalhães et al. [6] utilizaram a técnica de enxames de partículas para determinar as características biomecânicas da córnea. Ariza et al. [7], desenvolveram uma ferramenta preditiva para determinar propriedades mecânicas específicas do paciente do tecido corneano humano, esta ferramenta considera como variáveis de entrada a espessura central da córnea com uma pressão intra-ocular e a amplitude de deformação máxima da córnea, quando submetida a um teste de tonometria. Lago et al. [8] apresentaram uma nova metodologia para realizar a estimativa das constantes elásticas que caracterizam o comportamento biomecânico específico da córnea humana de determinados pacientes.

Vários estudos consideram a córnea como sendo material hiperelástico. O processo de caracterização de tecidos com comportamento hiperelástico tem tido um crescente interesse, em razão da necessidade de se obter um conhecimento profundo desse tipo de comportamento, em particular os tecidos humanos [9]. [10], considerou que a córnea possui comportamento hiperelástico, com baixa rigidez inicial, aumentando, gradualmente, sob níveis de tensão mais elevados. [11] também consideraram córnea possuindo o comportamento hiperelástico, e estudaram o comportamento mecânico da córnea com ceratocone. [12] consideraram que a córnea possui comportamento hiperelástico, sendo desenvolvido um novo modelo em 3-D para estudar as propriedades biomecânicas da córnea. Verifica-se que novas pesquisas na área, envolvendo a hiperelasticidade da córnea vêm sendo desenvolvidas, o que justifica a continuidade de estudos utilizando modelos hiperelásticos para esse fim.

Estudos computacionais envolvendo o comportamento hiperelástico de córneas podem auxiliar o tratamento de diversas doenças oculares, bem como no projeto e construção de equipamentos eficientes de diagnóstico, ou até mesmo, na melhoria de processos cirúrgicos [13], porém este tipo de estudo normalmente requer alto custo computacional. Sendo assim, o objetivo deste trabalho é o estudo do tempo de processamento para análises do comportamento mecânico da córnea, via simulações numéricas, utilizando modelos computacionais de baixa complexidade.

\section{Referencial Teórico}

A modelagem matemática da córnea consiste na representação numérica de uma parte do olho humano com o objetivo de prever comportamento mecânico por meio de simulações numéricas, sendo o Método dos Elementos Finitos (MEF), um dos mais utilizados para este fim. O MEF consiste na discretização de um meio contínuo em pequenos volumes, sendo mantidas as mesmas propriedades do meio original. Cada um desses pequenos volumes podem ser descritos por meio de equações diferenciais, para que, ao final do processo de simulações, os resultados sejam obtidos numericamente. O MEF pode ser utilizado nas diversas áreas das ciências exatas e biológicas, devido à sua grande aplicabilidade e eficiência na análise e coleta dos resultados [14].

A utilização de simulações numéricas, com o intuito de representação das propriedades deste tipo de material, pode ser realizada por meio dos princípios do MEF, possuindo vantagens sob o ponto de vista financeiro (por não necessitar de protótipos) e de custo computacional (tempo de processamento) para a análise e solução do problema abordado. O principal obstáculo na implementação dos métodos numéricos em estudos referentes aos materiais hiperelásticos é a busca por modelos numéricos robustos e confiáveis, que simulem o comportamento mecânico deste tipo de material [15].

Existem modelos numéricos utilizados especificamente para simulações envolvendo elementos finitos, por exemplo, o modelo de Ogden [16], o qual representa modelos fenomenológicos, objetivando a determinação da energia potencial de deformação. Nesses modelos hiperelásticos, tem-se a necessidade de determinar os coeficientes da energia potencial de deformação, que são calculados a partir de dados experimentais obtidos em ensaios mecânicos [17].

Ogden [18] propôs um modelo para materiais hiperelásticos, isotrópicos e incompressíveis, como pode ser observado em (1), derivando a função densidade de energia de deformação em termos das três extensões principais $\lambda_{p}, \mathrm{p}=1,2,3$. A função energia de deformação foi expandida por meio de uma série de potências reais e descrita como função dos estiramentos principais:

$$
\Psi=\sum_{p=1}^{N} \frac{\mu_{p}}{\alpha_{p}} *\left(\left(\lambda_{1}\right)^{\alpha_{p}}+\left(\lambda_{2}\right)^{\alpha_{p}}+\left(\lambda_{3}\right)^{\alpha_{p}}\right)-3
$$

Revista Brasileira de Computação Aplicada (ISSN 2176-6649), Passo Fundo, v. 9, n. 4, p. 32-42, dez. 2017 
onde $\Psi$ é a função energia de deformação, N é o número total de termos da série, $\mu_{p}$ e $\alpha_{p}$ são constantes do material, os parâmetros $\lambda_{1}, \lambda_{2}$ e $\lambda_{3}$ são os alongamentos.

O módulo de cisalhamento ( $\mu$ ) é descrito em (2), com a seguinte condição de estabilidade, $\mu_{n} \alpha_{n}>0$.

$$
\mu=\frac{1}{2} \sum_{p=1}^{N} \mu_{n} \alpha_{n}
$$

Além do MEF, outros métodos são aplicados para análise do comportamento biomecânico de córneas, como por exemplo, a técnica por enxame de partículas [6], métodos híbridos utilizando nanoindentação [19] e a técnica de microscopia de força atômica [20].

\section{Métodos}

Os dados do comportamento mecânico da córnea foram obtidos junto ao Laboratório de Biomecânica Ocular da Universidade de Liverpool. Durante o processo de configuração do experimento levou-se em consideração a correlação entre as propriedades mecânicas da córnea e da esclera. Para isto, um sistema de apoio do globo ocular foi desenvolvido, para que o mesmo pudesse flutuar em um meio de baixa rigidez, permitindo que toda a superfície ocular tenha apenas uma ligação rígida, aproximando-se do comportamento de apoio de um globo ocular real. Além disso, um método de monitoramente, baseado em câmeras de alta resolução digital e um software de processamento de imagens foram utilizados no intuito de se manter a precisão das medições realizadas. A Figura 1 representa o equipamento utilizado para a coleta dos dados experimentais e seus componentes.

Figura 1: Setup do teste de inflação de córneas [21] - 1- região na qual a córnea é fixada; 2- líquido responsável por promover a pressão no interior da córnea; 3 - medidor de temperatura; 4- fonte de laser; 5- câmeras CCD.

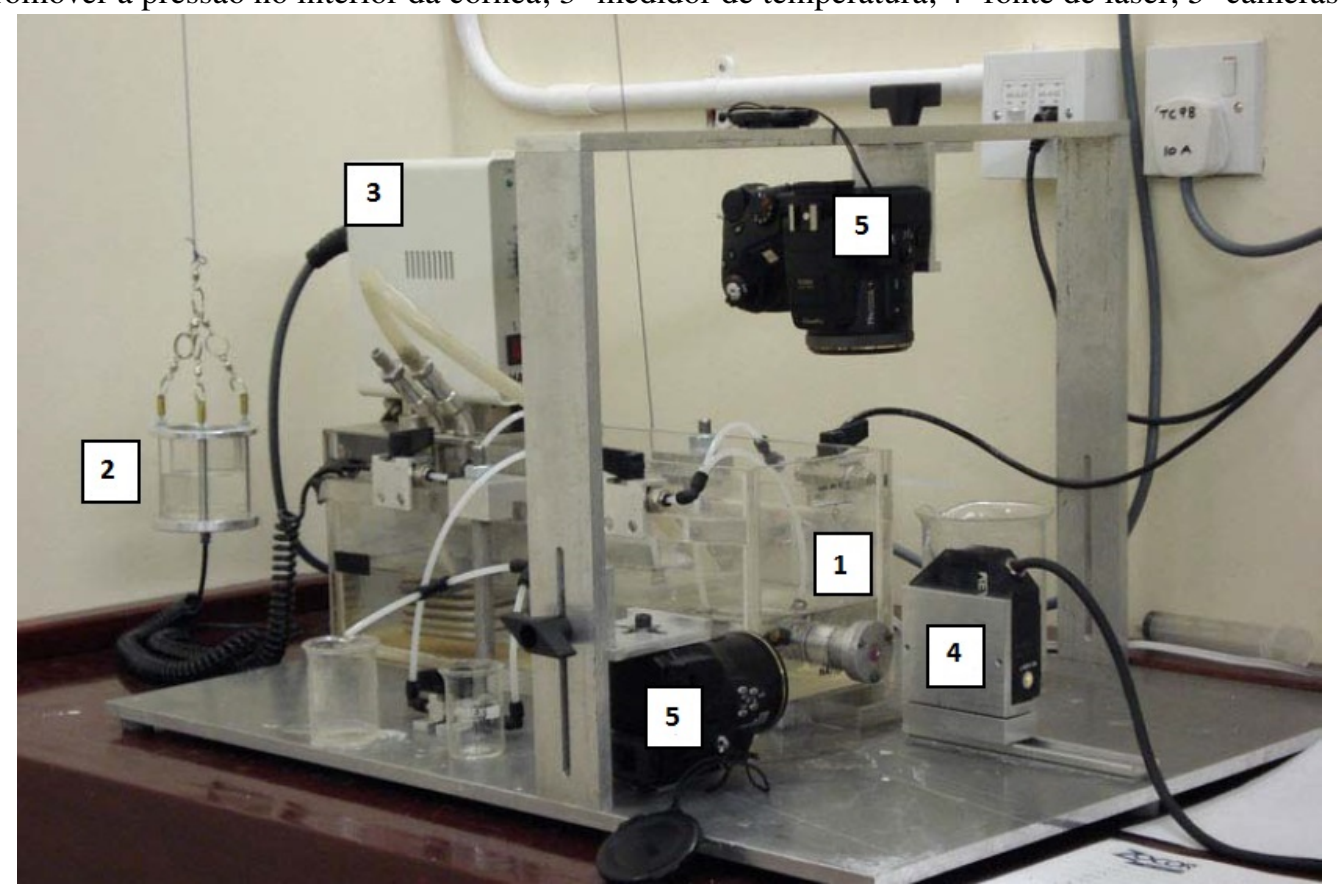

Nos experimentos, a córnea foi fixada e submetida a uma determinada pressão $(45 \mathrm{mmHg})$ aplicada por meio de um líquido contido em um reservatório específico. Um feixe de laser foi usado para a projeção de um ponto de referência na superfície da córnea. Imagens foram coletadas pelas câmeras CCD's e os deslocamentos foram obtidos por meio de processamento de imagens. A partir dos experimentos realizados, os deslocamentos no 
ponto central da córnea foram obtidos. A Figura 2 apresenta os valores experimentais obtidos (aqui denominado por target). Estes dados foram coletados utilizando-se 21 variações de pressão em um intervalo de 0 a $45 \mathrm{mmHg}$.

Figura 2: Representação dos dados experimentais - (target)

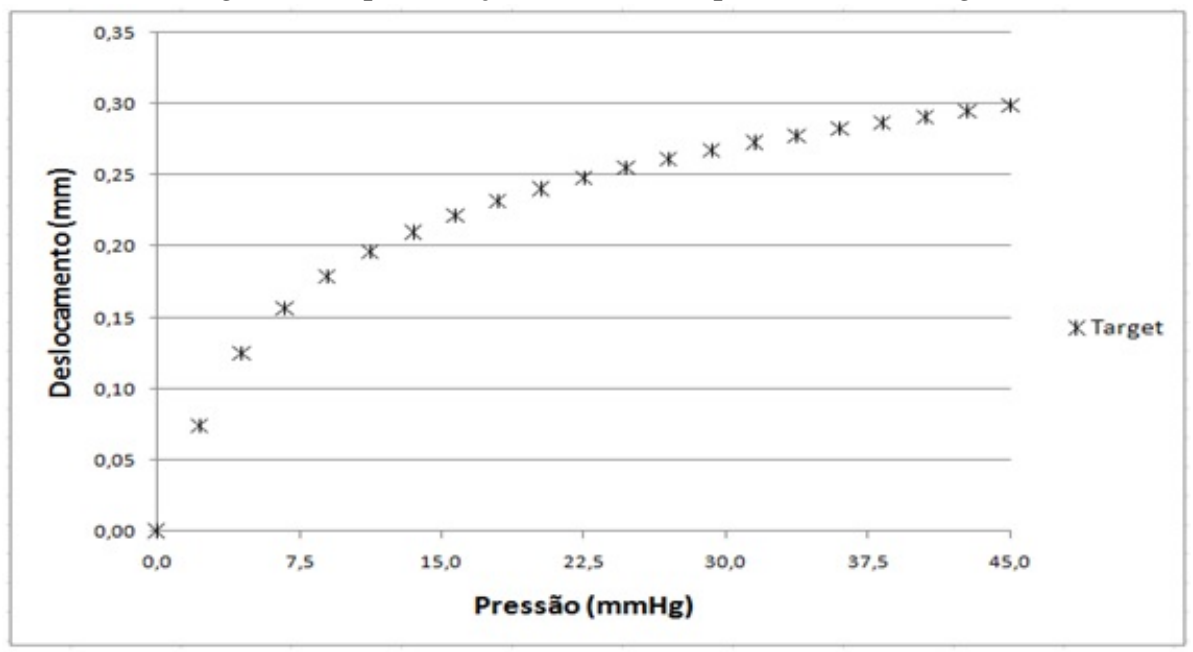

As simulações ocorreram seguindo os mesmos parâmetros experimentais, ou seja, a geometria da córnea foi fixada nas extremidades, sendo que a mesma foi deformada a partir dos esforços internos. Neste caso, a geometria da córnea foi discretizada formando uma malha de elementos finitos. A Figura 3 mostra a geometria de uma córnea, levando-se em consideração a fixação e a região de aplicação da carga para as simulações.

Figura 3: Geometria discretizada de uma córnea para fins de simulação numérica.

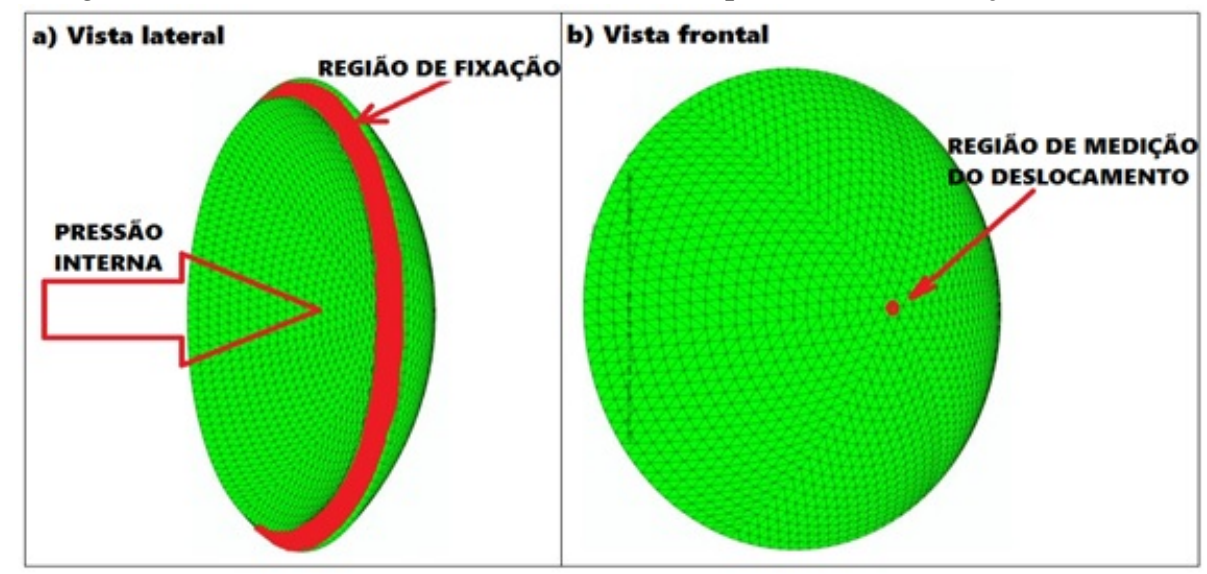

Para gerar modelos computacionais da córnea com baixa complexidade (modelos com número de nós inferior a 1000), foi utilizado um programa desenvolvido no laboratório de biomecânica da Universidade de Liverpool. Além disso, as constantes do material ( $\mu$ e $\alpha$ ) foram obtidas por meio do software comercial HEEDS, que buscou otimizar os melhores valores destas constantes para representar o material, a partir da curva gerada pelos experimentos (Figura 2).

Para simular os deslocamentos na córnea, utilizou-se o software comercial de elementos finitos Abaqus ${ }^{\circledR}$, em conjunto com outros algoritmos executáveis. O algoritmo principal tem a função de deletar os arquivos temporários e executar o software Abaqus ${ }^{\circledR}$ para gerar um arquivo contendo os resultados (extensão .ODB). A partir daí, este algoritmo principal executa um outro algoritmo, desenvolvido em linguagem Python, também na Universidade de Liverpool, para extrair os deslocamentos em um arquivo de texto. O processo de execução deste algoritmo 
principal está apresentado no fluxograma da Figura 4.

Figura 4: Fluxograma do algoritmo principal usado para gerar os deslocamentos simulados na córnea.

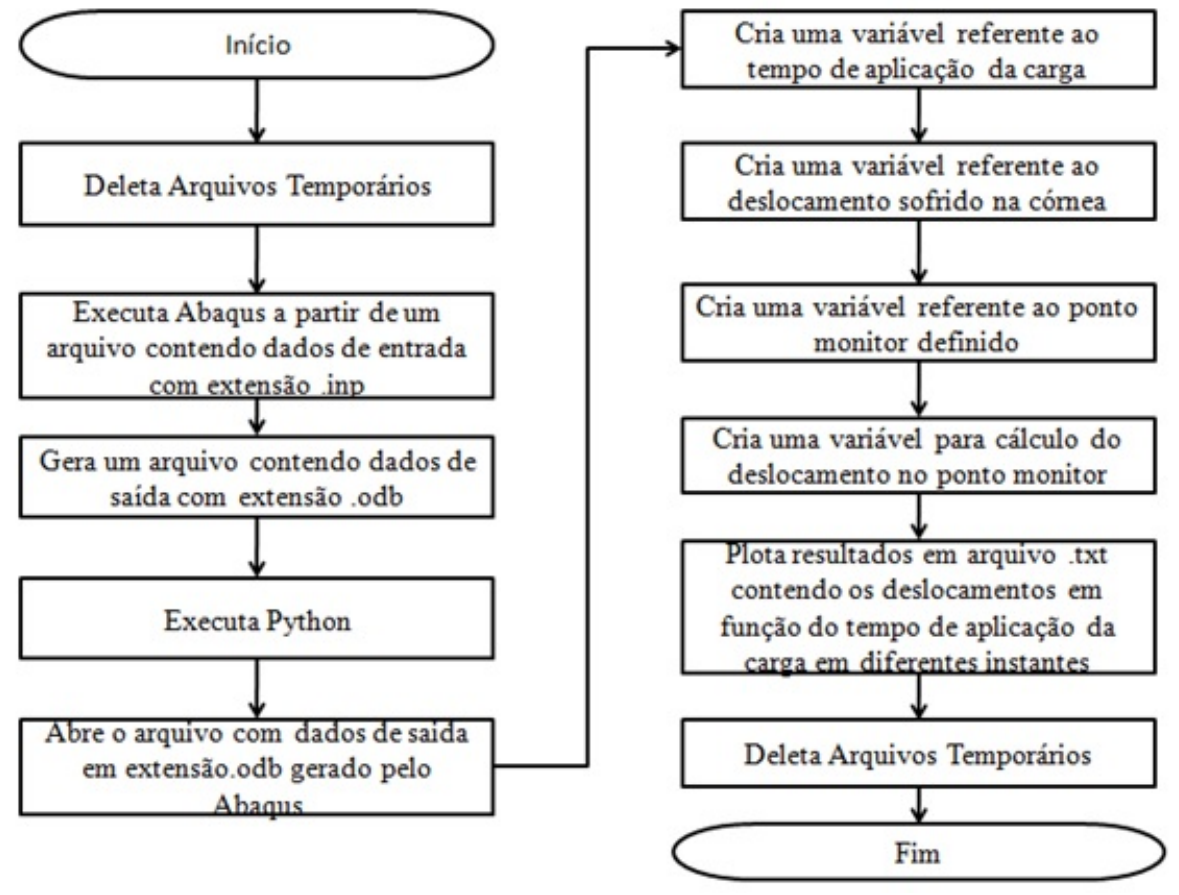

\subsection{Cenários Simulados}

Foram realizadas 9 variações nas quantidades de nós e elementos dos modelos computacionais de córneas, o que gerou 9 cenários diferentes para análises. Essas variações estão relacionadas as quantidades de anéis e layers da malha de elementos finitos, as quais representam a estrutura de uma córnea.

As figuras 5 e 6, representam, como exemplos, duas geometrias geradas por meio de variações do número de anéis e layers que compõem a discretização da córnea. Consequentemente, obtiveram-se variações nas quantidades de nós e elementos das respectivas geometrias.

As informações de todos os cenários simulados estão apresentadas, de maneira resumida, na Tabela 1:

Tabela 1: Informações das Simulações Realizadas

\begin{tabular}{ll}
\hline Cenários & Qtde de Nós e Elementos \\
\hline 1 & 57 Nós e 48 Elementos (6 nós por elemento) \\
2 & 74 Nós e 54 Elementos (6 nós por elemento) \\
3 & 291 Nós e 54 Elementos (15 nós por elemento) \\
4 & 111 Nós e 108 Elementos (6 nós por elemento) \\
5 & 455 Nós e 108 Elementos (15 nós por elemento) \\
6 & 182 Nós e 150 Elementos (6 nós por elemento) \\
7 & 753 Nós e 150 Elementos (15 nós por elemento) \\
8 & 254 Nós e 216 Elementos (6 nós por elemento) \\
9 & 938 Nós e 864 Elementos (6 nós por elemento) \\
\hline
\end{tabular}

Revista Brasileira de Computação Aplicada (ISSN 2176-6649), Passo Fundo, v. 9, n. 4, p. 32-42, dez. 2017 
Figura 5: Geometria formada por 2 anéis e 2 layers, contendo 57 nós e 48 elementos.

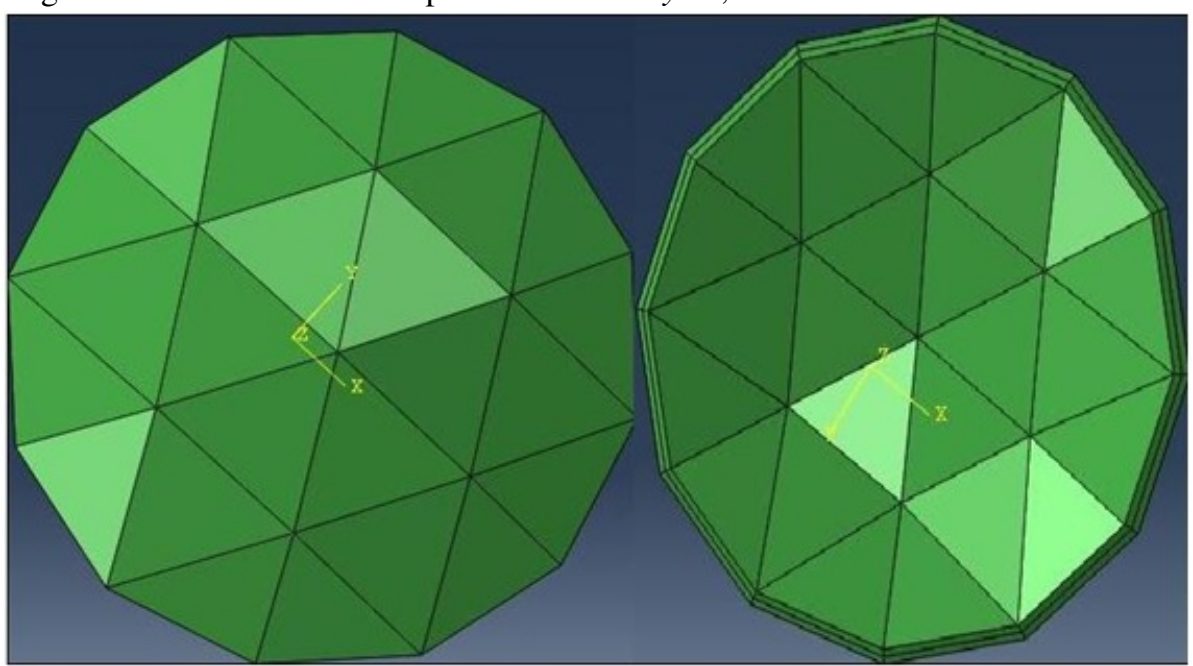

Figura 6: Geometria formada por 12 anéis e 1 layer contendo 938 nós e 864 elementos.

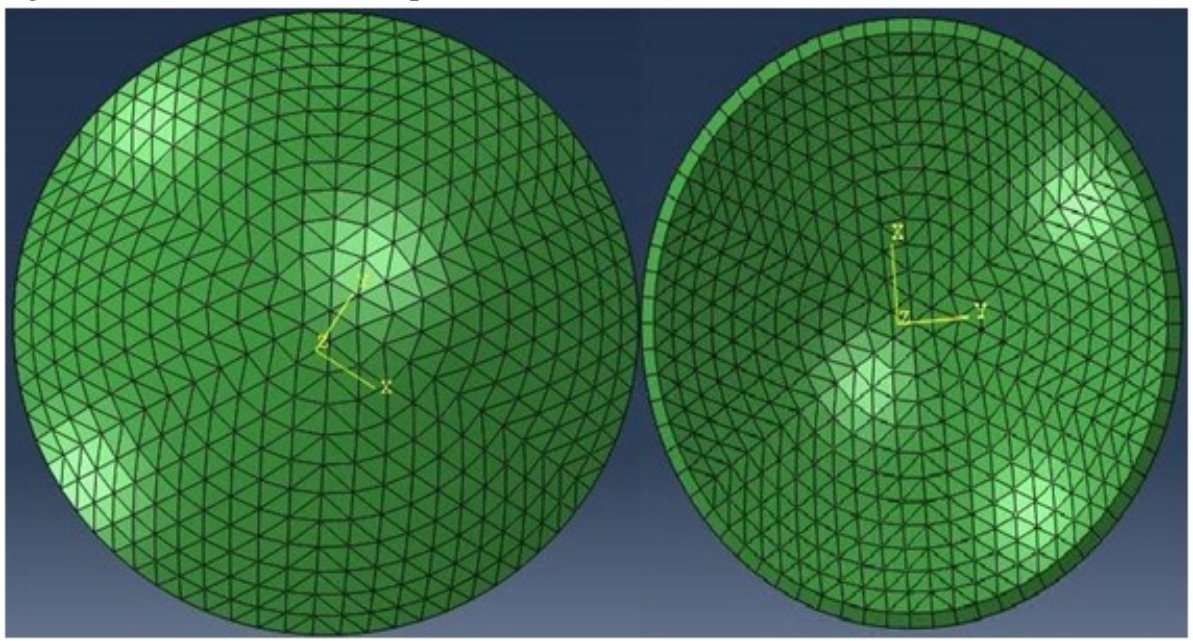

\section{Resultados e Discussão}

\subsection{Tempo de Processamento dos Cenários Simulados}

As informações dos tempos de processamento dos cenários simulados estão apresentadas na Figura 7. A configuração do equipamento utilizado para a realização dos testes de desempenho possui processador AMD Phenom II X4B93, memória RAM de 4 Gb e placa de vídeo Evga Gt 730.

Nota-se por meio da Figura 7 que os cenários 1 (57 nós) e 2 (74 nós) possuem os menores tempos de processamento. Esse fato se deve, evidentemente, ao menor tempo de processamento das simulações para modelos com menor número de nós.

No intuito de se verificar a precisão dos resultados simulados, foi realizada a comparação dos deslocamentos virtuais obtidos no software Abaqus ${ }^{\circledR}$ e o descolamento real, obtido experimentalmente. A Figura 8 apresenta os resultados dos cenários 1 a 4 (Tabela 1), quando comparado ao target.

Nota-se na Figura 8, que no cenário 3, composto por 291 nós, obteve-se resultado satisfatório, visto que os 
Figura 7: Tempo de Processamento dos Cenários Simulados.

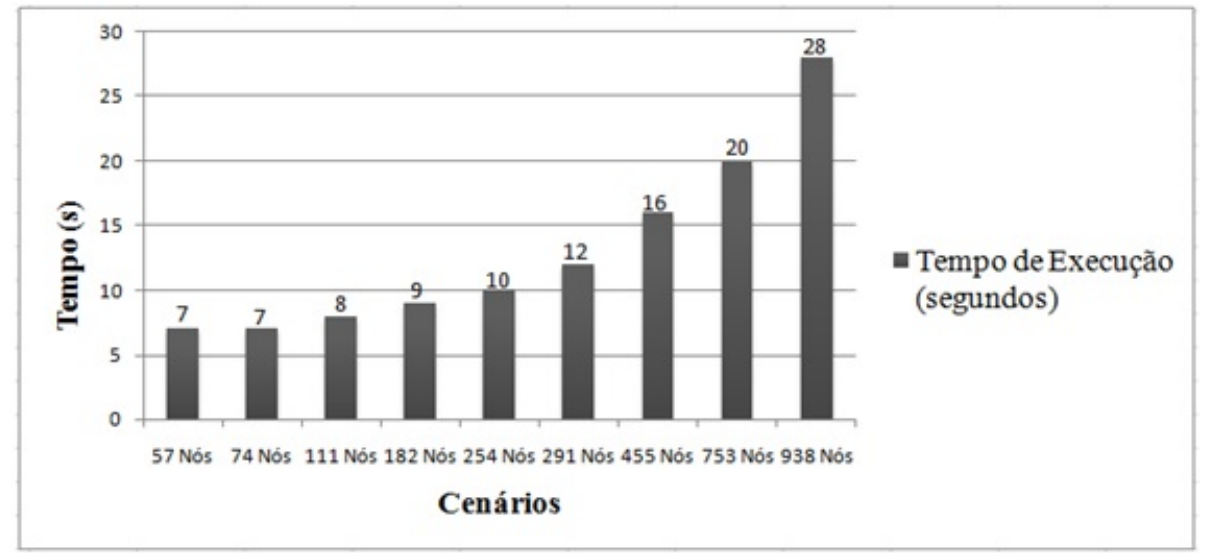

Figura 8: Deslocamento obtido x Target para os cenários de 1 a 4.

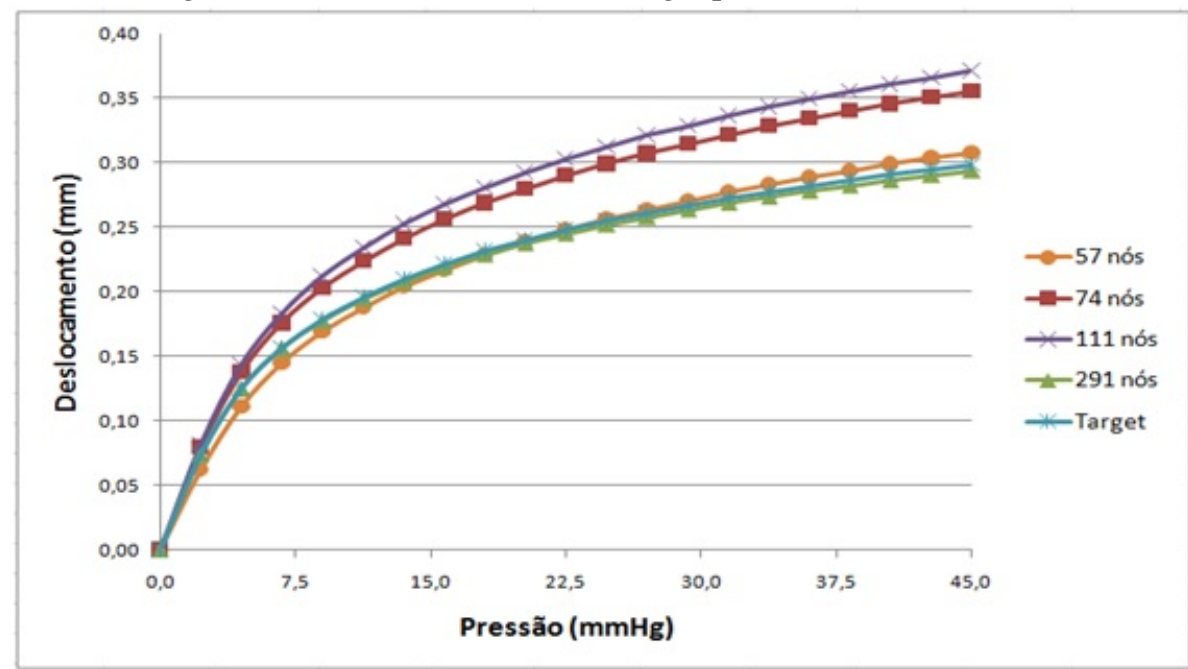

deslocamentos simulados para essa geometria foram os que mais se aproximaram do target.

A Figura 9 apresenta os resultados dos cenários 5 a 9 (Tabela 1), quando comparado ao target.

Verifica-se na Figura 9, que o cenário 5, composto de 455 nós, também obteve resultado satisfatório, ou seja, próximo ao target. No intuito de se verificar qual cenário simulado obteve melhor comportamento, quando comparado aos dados experimentais (target), calculou-se a diferença por meio da soma dos erros ao quadrado.

\subsection{Soma dos erros ao quadrado}

A diferença entre cenários simulados e o target foi obtida por meio da comparação entre os valores de deslocamento simulados no software Abaqus ${ }^{\circledR}$ e os dados experimentais, como pode ser observado em (3), a qual representa a soma dos erros ao quadrado (SSE)

$$
S S E=\frac{1}{n} \sum_{i=0}^{n} \sqrt{\left(x_{i}-y_{i}\right)^{2}}
$$


Figura 9: Deslocamento Obtido x Target para os cenários de 5 a 9.

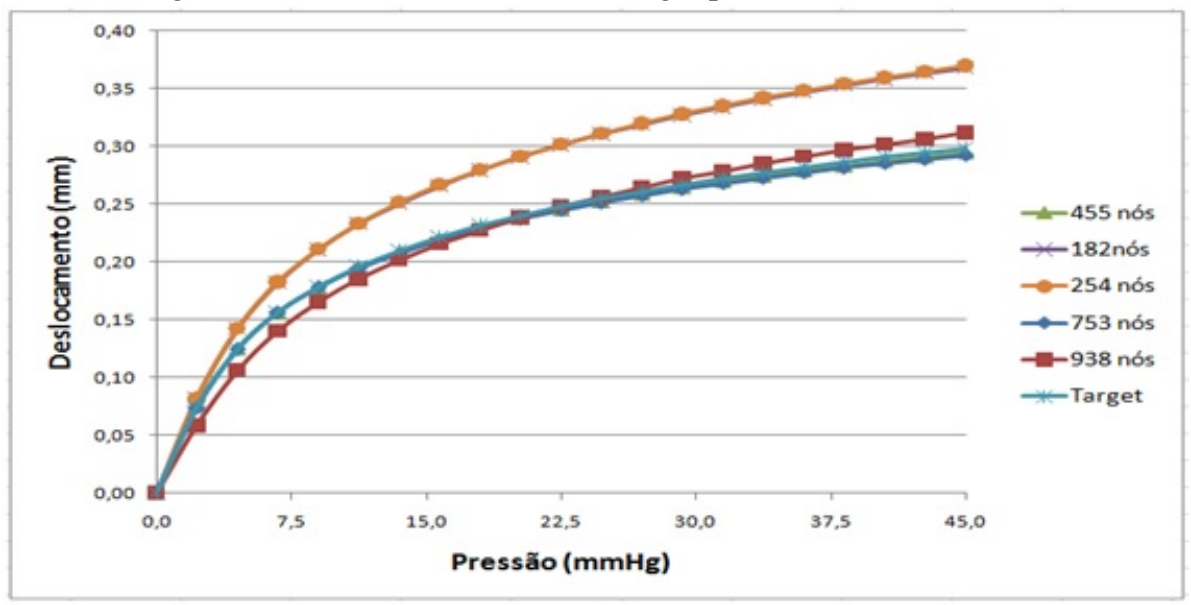

onde x representa o valor do deslocamento obtido no software Abaqus ${ }^{\circledR}$, y representa o valor do target e $\mathrm{n}$ corresponde à variação de pressão.

Realizou-se a análise da soma dos erros ao quadrado, conforme Figura 10.

Figura 10: Resultado de SSE para os cenários simulados.

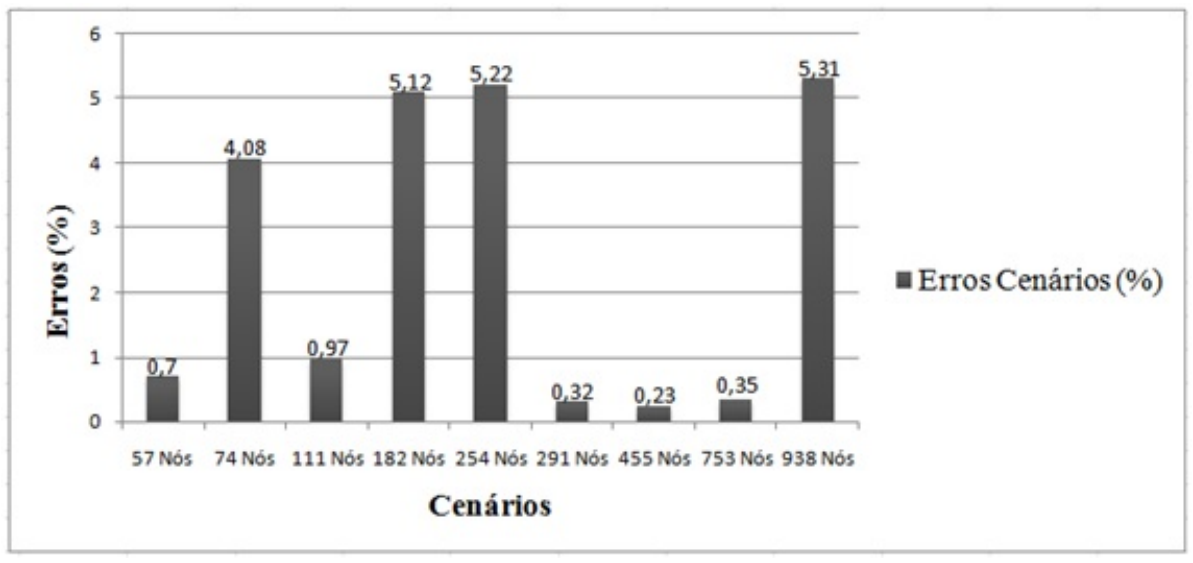

A partir da Figura 10, conclui-se que o menor valor de SSE (0,23\%) foi obtido para a geometria representada pelo cenário de 455 nós. A Tabela 2 apresenta o tempo de processamento e o SSE para todos os cenários simulados.

Como pode ser verificado na Tabela 2, nem sempre o menor tempo de processamento ocorre para cenários com menor SSE. Um exemplo disto pode ser observado no cenário 5 (455 nós), que gerou o menor valor de SSE $(0,23 \%)$, quando comparado aos demais cenários, porém as simulações mostraram um tempo de processamento mediano (16 s). Já no cenário 2 (74 nós), o tempo de processamento foi de $7 \mathrm{~s}$, porém nas simulações, este cenário obteve um valor de SSE igual a 4,08 \%, o que implica em menor precisão dos resultados simulados.

\subsection{Simulações variando as idades}

No intuito de se comprovar, por simulações, que os menores deslocamentos na córnea ocorrem em pacientes com idades mais avançadas, utilizou-se como referência o cenário com 182 nós para simular o deslocamento de córneas. A Figura 11 apresenta a variação do deslocamento com a variação das idades para esse cenário. 
Tabela 2: Informações das Simulações Realizadas

\begin{tabular}{lll}
\hline Cenários & Tempo de processamento (s) & SSE \\
\hline 1 (57 nós) & 7 segundos & $0,70 \%$ \\
2 (74 Nós) & 7 segundos & $4,08 \%$ \\
3 (291 nós) & 12 segundos & $0,32 \%$ \\
4 (111 nós) & 8 segundos & $0,97 \%$ \\
5 (455 nós) & 16 segundos & $0,23 \%$ \\
6 (182 nós) & 9 segundos & $5,12 \%$ \\
7 (753 nós) & 20 segundos & $0,35 \%$ \\
8 (254 nós) & 10 segundos & $5,22 \%$ \\
9 (938 nós) & 28 segundos & $5,31 \%$ \\
\hline
\end{tabular}

Figura 11: Variação do deslocamento da córnea em função da idade do paciente.

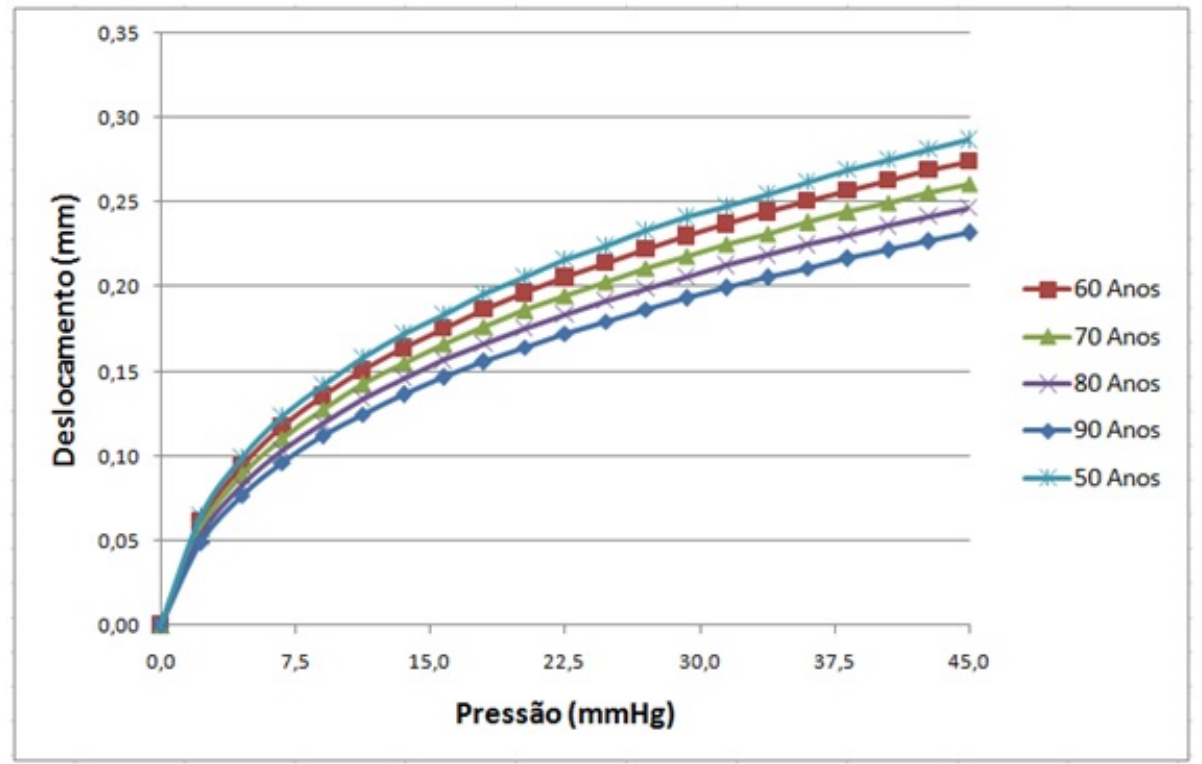

Ao realizar as variações nas idades dos pacientes para o cenário simulado, conclui-se que, com o aumento da idade do paciente, tem-se um aumento da rigidez da córnea, reforçando a hipótese de que os resultados das simulações são confiáveis.

\section{Conclusões}

Neste trabalho, foram desenvolvidos modelos computacionais para estudo do comportamento mecânico da córnea humana via simulações numéricas, admitindo comportamento hiperelástico. Foram realizadas variações na quantidade de nós e elementos de cada um desses modelos. Nas simulações, considerou-se o modelo de Ogden (Equação 1), em que os resultados foram comparados aos deslocamentos obtidos em experimentos realizados na Universidade de Liverpool.

A partir dos resultados gerados, observou-se que o menor valor de SSE $(0,23 \%)$ foi obtido para um modelo geométrico de córneas com 455 nós, ou seja, dentro dos limites encontrados na literatura para simulações usando número de nós inferior a 1000 [22]. Observou-se ainda que o tempo de processamento é um fator importante em simulações e deve ser levado em consideração. Por exemplo, no cenário de 938 nós, o tempo de processamento foi de $28 \mathrm{~s}$, que está próximo ao tempo de processamento obtido em [23] para simulações utilizando número de nós superiores a 1000 , porém com erros superiores a $10 \%$. 
Caso necessário utilizar um conjunto de simulações com 1000 interações para fins de otimização, seriam necessárias 7,8 h, ao passo que, se fosse utilizado o cenário com 57 nós (menor tempo encontrado, 7 segundos), seria necessária 1,95 h de simulação. No entanto, verificou-se ainda que, nem sempre o menor tempo de processamento ocorre para cenários com maior precisão. Isto porque, o menor tempo de processamento não necessariamente ocorre para cenários com maior precisão, e sim para cenários com menor número de nós.

Ainda diante dos resultados obtidos, comprovou-se por simulações que, com o aumento da idade do paciente, tem-se um aumento da rigidez da córnea. Com tudo isso, conclui-se que estudos a respeito do comportamento mecânico de córneas via simulações numéricas é de fundamental importância para a área oftalmológica. Portanto, este estudo pode ser útil para tratamentos oftalmológicos por meio de simulações numéricas que requerem resposta rápida em termos de processamento computacional.

\section{Agradecimentos}

À Universidade de Liverpool e à CAPES pelo apoio financeiro durante a realização desse projeto.

\section{Referências}

[1] BOOTE, C. et al. Lamellar orientation in human cornea in relation to mechanical properties. Journal of structural biology, Elsevier, v. 149, n. 1, p. 1-6, 2005. Disponível em: <https://doi.org/10.1016/j.jsb.2004. 08.009>. Acesso em: 28 abr. 2017.

[2] HAMILTON, K. E.; PYE, D. C. Young's modulus in normal corneas and the effect on applanation tonometry. Optometry \& Vision Science, LWW, v. 85, n. 6, p. 445-450, 2008. Disponível em: <https://journals.lww.com/optvissci/Abstract/2008/06000/Young_s_Modulus_in_Normal_Corneas_ and_the_Effect.15.aspx>. Acesso em: 28 abr. 2017.

[3] LEANDRO, D. d. C. Avaliação biomecânica de córneas de suínos por meio da microscopia de força atômica. Dissertação (Mestrado em Ciências) — Universidade de São Paulo, São Paulo, 2010.

[4] KOTECHA, A. What biomechanical properties of the cornea are relevant for the clinician? Survey of ophthalmology, Elsevier, v. 52, n. 6, p. S109-S114, 2007. Disponível em: <https://doi.org/10.1016/j.survophthal. 2007.08.004>. Acesso em: 28 abr. 2017.

[5] HJORTDAL, J. Ø. Regional elastic performance of the human cornea. Journal of biomechanics, Elsevier, v. 29, n. 7, p. 931-942, 1996. Disponível em: <https://doi.org/10.1016/0021-9290(95)00152-2>. Acesso em: 28 abr. 2017.

[6] MAGALHÃES, R. et al. Application of particle swarm optimization in inverse finite element modeling to determine the cornea's mechanical behavior. Acta Scientiarum: Technology, v. 39, n. 3, 2017. Disponível em: <http://dx.doi.org/10.4025/actascitechnol.v39i3.29884>. Acesso em: 31 out. 2017.

[7] ARIZA-GRACIA, M. Á. et al. A predictive tool for determining patient-specific mechanical properties of human corneal tissue. Computer Methods in Applied Mechanics and Engineering, Elsevier, v. 317, p. 226247, 2017. Disponível em: <https://doi.org/10.1016/j.cma.2016.12.013>. Acesso em: 31 out. 2017.

[8] LAGO, M. et al. A new methodology for the in vivo estimation of the elastic constants that characterize the patient-specific biomechanical behavior of the human cornea. Journal of biomechanics, Elsevier, v. 48, n. 1, p. 38-43, 2015. Disponível em: <https://doi.org/10.1016/j.jbiomech.2014.11.009>. Acesso em: 31 out. 2017.

[9] AFONSO, J. et al. Mechanical properties of polypropylene mesh used in pelvic floor repair. International urogynecology journal, Springer, v. 19, n. 3, p. 375-380, 2008. Disponível em: <https://link.springer.com/ article/10.1007/s00192-007-0446-1>. Acesso em: 28 abr. 2017.

Revista Brasileira de Computação Aplicada (ISSN 2176-6649), Passo Fundo, v. 9, n. 4, p. 32-42, dez. 2017 
[10] ELSHEIKH, A. Finite element modeling of corneal biomechanical behavior. Journal of Refractive Surgery, Slack Incorporated, v. 26, n. 4, p. 289-300, 2010. Disponível em: <https://doi.org/10.3928/ 1081597X-20090710-01>. Acesso em: 28 abr. 2017.

[11] HAN, Z. et al. Biomechanical and refractive behaviors of keratoconic cornea based on three-dimensional anisotropic hyperelastic models. Journal of Refractive Surgery, SLACK Incorporated, v. 29, n. 4, p. 282290, 2013. Disponível em: <https://doi.org/10.3928/1081597X-20130318-08>. Acesso em: 28 abr. 2017.

[12] ROY, A. S. et al. Air-puff associated quantification of non-linear biomechanical properties of the human cornea in vivo. Journal of the mechanical behavior of biomedical materials, Elsevier, v. 48, p. 173-182, 2015. Disponível em: <https://doi.org/10.1016/j.jmbbm.2015.04.010>. Acesso em: 28 abr. 2017.

[13] HAMILTON, K. E.; PYE, D. C. Young's modulus in normal corneas and the effect on applanation tonometry. Optometry \& Vision Science, LWW, v. 85, n. 6, p. 445-450, 2008. Disponível em: <https://10.1097/OPX. 0b013e3181783a70>. Acesso em: 28 abr. 2017.

[14] LOTTI, R. S. et al. Aplicabilidade científica do método dos elementos finitos. R Dental Press Ortodon Ortop Facial, SciELO Brasil, v. 11, n. 2, p. 35-43, 2006. Disponível em: <http://www.scielo.br/pdf/dpress/v11n2/ a06v11n2>. Acesso em: 28 abr. 2017.

[15] RIBEIRO, J. et al. Caracterização experimental e numérica do campo de deslocamentos de tecidos biológicos hiper-elásticos. In: X Congresso Ibero-Americano em Engenharia Mecânica. [s.n.], 2011. p. 2603-2609. Disponível em: <http://hdl.handle.net/10198/9824>. Acesso em: 28 abr. 2017.

[16] TABER, L. A. Nonlinear theory of elasticity: applications in biomechanics. Estados Unidos: World Scientific, 2004.

[17] MARTINS, P.; JORGE, R. N.; FERREIRA, A. A comparative study of several material models for prediction of hyperelastic properties: Application to silicone-rubber and soft tissues. Strain, Wiley Online Library, v. 42, n. 3, p. 135-147, 2006. Disponível em: <https://doi.org/10.1111/j.1475-1305.2006.00257.x>. Acesso em: 28 abr. 2017.

[18] OGDEN, R.; SACCOMANDI, G.; SGURA, I. Fitting hyperelastic models to experimental data. Computational Mechanics, Springer, v. 34, n. 6, p. 484-502, 2004. Disponível em: <https://doi.org/10.1007/ s00466-004-0593-y>. Acesso em: 28 abr. 2017.

[19] ABYANEH, M. et al. A hybrid approach to determining cornea mechanical properties in vivo using a combination of nano-indentation and inverse finite element analysis. Journal of the mechanical behavior of biomedical materials, Elsevier, v. 27, p. 239-248, 2013. Disponível em: <https://doi.org/10.1016/j.jmbbm.2013. 05.016>. Acesso em: 31 out. 2017.

[20] DEVI, A. L.; NONGTHOMBA, U.; BOBJI, M. Quantitative characterization of adhesion and stiffness of corneal lens of drosophila melanogaster using atomic force microscopy. journal of the mechanical behavior of biomedical materials, Elsevier, v. 53, p. 161-173, 2016. Disponível em: <https://doi.org/10.1016/j.jmbbm. 2015.08.015>. Acesso em: 31 out. 2017.

[21] ELSHEIKH, A. Understanding corneal biomechanics through experimental assessment and numerical simulation. Estados Unidos: Nova Science Publishers, 2010.

[22] PASZYŃSKA, A. et al. Linear computational cost graph grammar based direct solver for 3d adaptive finite element method simulations. IJMMM International Journal of Materials, Mechanics and Manufacturing, v. 1, p. 225-230, 2013. Disponível em: <https://doi.org/10.7763/IJMMM.2013.V1.48>. Acesso em: 31 out. 2017.

[23] FARMAGA, I. et al. Evaluation of computational complexity of finite element analysis. In: IEEE. CAD Systems in Microelectronics (CADSM), 2011 11th International Conference The Experience of Designing and Application of. 2011. p. 213-214. Disponível em: <http://ieeexplore.ieee.org/abstract/document/5744437/>. Acesso em: 31 out. 2017. 